\title{
Nitric oxide mediated mechanisms adopted by plants to cope with salinity
}

\author{
A. SHARMA ${ }^{1 *}$, D. KAPOOR ${ }^{2}$, J. WANG ${ }^{1}$, M. LANDI ${ }^{3}$, B. ZHENG ${ }^{1}$, D. YAN ${ }^{1}$, and H. YUAN ${ }^{1 *}$ \\ State Key Laboratory of Subtropical Silviculture, Zhejiang A\&F University, Hangzhou 311300, P.R. China ${ }^{1}$ \\ School of Bioengineering \& Biosciences, Lovely Professional University, Punjab 144411, India ${ }^{2}$ \\ Department of Agriculture, Food and Environment, University of Pisa, 56124 Pisa, Italy ${ }^{3}$
}

\begin{abstract}
Worldwide, a relevant surface of arable lands is facing salt stress, and this surface is increasing continuously due to both natural and anthropogenic activities. Nitric oxide (NO) is a small, gaseous molecule with a plethora of physiological roles in plants. In addition to its normal physiological functions, NO protects plants subjected to different environmental cues including salinity. For example, NO mediates photosynthesis and stomatal conductance, stimulates the activity of $\mathrm{Na}^{+} / \mathrm{H}^{+}$antiport in tonoplast, promotes the biosynthesis of osmolytes, and counteracts overaccumulation of reactive oxygen species in plant cells under salt stress. Exogenous NO is also beneficial for plants subjected to salinity, in which it increases salinity tolerance via growth promotion, reversing oxidative damage, and maintaining ion homeostasis. This review provides a comprehensive picture of the NO-mediated mechanisms in plants, resulting in salinity tolerance with a particular focus on the photosynthetic processes, the antioxidant patterns as well as the cross-talk with other regulatory compounds in plant cells.
\end{abstract}

Additional key words: abiotic stresses, antioxidant systems, osmolytes, photosynthesis, stomatal conductance.

\section{Introduction}

The increasing trend of population growth paralleled, unfortunately, the rate of water and soil salinization, posing serious concerns in a near future for the World's food production. Good quality water and soil is the prerequisite for a high yield of the plants. Conversely, one of the most deleterious phenomena, in particular in arid and semi-arid areas, is salinization of water and soils (Rui and Ricardo 2017). During the last century a huge number of aquifers and river basins have become unsuitable for human consumption owing to high salinity. Moreover, every year a large fraction of agricultural land is salinized and becomes unusable (Vengosh 2003). So, in order to enhance crop yield, it becomes necessary to find new strategies for better plant performance in saline conditions

\section{(Hanin et al. 2016).}

Nitric oxide (NO) is a redox-signaling molecule involved in many physiological processes in plants, and plays key roles in response to challenging growth conditions, including salinity (Zhao et al. 2004, Zhang et al. 2006b). In particular, it has been demonstrated that NO can enhance salt tolerance through increasing activities of proton-pump and $\mathrm{Na}^{+} / \mathrm{H}^{+}$antiport in the tonoplast (Zhang et al. 2006b). In addition, NO is itself a reactive species and can be either protective or toxic depending to several factors including concentration, the plant species, and the plant developmental stages (Zhao et al. 2007). Nitric oxide may therefore act as a chain breaker to minimize the oxidative damage attributable to salttriggered oxidative stress (Zhao et al. 2007, Gadelha et al. 2017). This signaling molecule also interacts with other

\footnotetext{
Submitted 25 February 2020, last revision 20 April 2020, accepted 11 May 2020.

Abbreviations: ABA - abscisic acid; BADH - betainealdehyde dehydrogenase; CAT - catalase; GB - glycine betaine; $g_{s}$ - stomatal conductance; GSH - glutathione; MDA - malondialdehyde; MAPK - mitogen-activated protein kinase; P5CS1 - pyrroline-5-carboxylate synthetase; $\mathrm{P}_{\mathrm{N}}$ - net photosynthetic rate; POD - peroxidase; ROS - reactive oxygen species; SNP - sodium nitroprusside; SOD superoxide dismutase.

Acknowledgements: This study was supported by the National Key Research and Development Program of China (2018YFD1000604), the Key Project of Zhejiang Provincial Natural Science Foundation (LZ18C160001), the National Natural Science Foundation of China $(31,901,346,31,971,695)$, and the Overseas Expertise Introduction Project for Discipline Innovation (111 Project D18008). The first three authors contributed equally to this work.

* Corresponding authors; e-mails: anketsharma@gmail.com,hwyuan@zafu.edu.cn
} 
radicals, antioxidant compounds, and phytohormones taking part to an orchestrated cross-talk between NO and other molecules against salinity (Hasanuzzaman et al. 2018). For example, NO mitigates salt stress by regulating amount of osmolytes and antioxidant enzymes in chickpea plants (Ahmad et al. 2016). All these aspects suggest that NO is a key metabolite in plant physiology and a depth knowledge of the mechanisms underpinning NO involvement against salinity are of crucial importance to promote the cultivation of plants in marginal area prone to salinity. The present literature survey aims at summarizing the main findings about the physiological roles of NO in plants suffering of salt stress with the scope to provide a comprehensive picture related to the role of this gaseous molecule under salinity, which could be useful for future research on the topic.

\section{Salt stress and its impacts on plants}

Salt stress is one of the most predominating abiotic stresses which lead to drastic loss in agriculture in terms of crop yield, majorly in dry and semi dry areas. Hence it is required to understand the various strategies which help in combating salinity stress and developing salt resistant plant cultivars (Tanveer et al. 2018). Deposition of excessive concentrations of salts leads to development of initial stress symptoms in the roots: osmotic stress. In the cytosol, long exposure of salt stress causes imbalance of minerals and nutrients. Salt stress consequently leads to oxidative burst due to the generation of reactive oxygen species (ROS) (Acosta-Motos et al. 2017, Li et al. 2018, Rattan et al. 2020). Additionally, uptake and accumulation of excessive salt ions interference with many intracellular metabolic processes. High salt concentration in the soil causes osmotic imbalance which limits water uptake from soil (Munns 2002). High concentration of $\mathrm{Na}^{+}$inhibits the absorption of some nutrient elements. Meanwhile, ionic and osmotic stress will also lead to an imbalance in plant metabolism and above mentioned oxidative stress (Chinnusamy et al. 2006). The salt stress decrease the production capacity due retardation in growth and finally can cause the death of the plant. Salt stress adversely disturbs virtually all facets of biological processes, including photosynthesis, protein synthesis, and other primary metabolism pathways (Chartzoulakis and Klapaki 2000, Tanveer et al. 2018, Rattan et al. 2020). Additionally, under high salt, the most immediate response is the decrease in the cell expansion (Wang and Nii 2000). Parida and Das (2005) showed that salinity disturbs plant photosynthesis by negatively affecting carbon assimilation efficiency. Thylakoid structure of chloroplasts also gets disrupted under salt stress accompanied by reduction in the starch content (Hernández et al. 2002). The chloroplasts were found to be aggregated together in the leaves of salt treated tomato. Moreover, no or very low grana and thylakoid structures were observed in chloroplasts (Khavari-Nejad and Mostofi 1998).

\section{Physiological roles of NO in plants}

Nitric oxide stimulates development of plants by regulating various growth parameters of primary root, hypocotyl, mesocotyl, adventitious and lateral roots, leaves, and the stem (Neil et al. 2008). For example, at low concentrations of NO donor nitroprusside, an acceleration of growth was noted for Arabidopsis thaliana primary root, whereas inhibition of growth was observed at higher concentrations (Yemets et al. 2009). It is established that NO participates in differentiation of Zinnia elegans xylem by regulating cell lignification and the programmed cell death (Ferrer and Ros Barceló 1999). In case of pathogen attack, NO also regulates plant defense system by modulating programmed cell death (Mur et al. 2005).

It is suggested that plants with elevated production of NO enter the flowering stage somewhat later than plants with physiologically normal NO content (He et al. 2004). Germinating and even non-germinating pollen grains produce NO and nitrate required for the reproduction process. The NO generation is possibly an important component of the signaling system activated by pollenstigma interaction. Nitric oxide regulates the direction of pollen tube growth, which is very important for fertilization. It was found that interaction of NO produced by the pollen with ROS generated by the pistil stigma can initiate pollination through the onset of signaling cascades between pollen grains and stigmas (Bright et al. 2009). In mutant Arabidopsis plants (Atnos1) with defective NO synthase, reduction in NO biosynthesis was observed accompanied by declined plant growth and stomatal movement. However, overexpression of AtNOS1 resulted in recovery of plant growth due to NO mediated regulation of plant biology (Guo et al. 2003), suggesting roles of this gaseous molecule in plant growth and development. In addition to normal physiological functions, NO regulates plant growth and development under abiotic stresses (Fancy et al. 2017, Sharma et al. 2020).

\section{Nitric oxide and its physiological roles in plants under salt stress}

Nitric oxide plays key roles in mitigating harmful effects of salinity in plants by regulating key physiological processes like photosynthesis, antioxidative defense system and ion homeostasis. The detailed description about NO mediated regulation of plant biology under salt stress is discussed in following sub-sections. The roles of $\mathrm{NO}$ in plants under salt stress are summarized in Table 1.

Regulation of photosynthesis: Salt stress severely impact the photosynthetic processes by decreasing chloroplast activity, and hampering photosynthetic rate $\left(\mathrm{P}_{\mathrm{N}}\right)$, and stomata conductance $\left(\mathrm{g}_{\mathrm{s}}\right)$ (Teixeira and Pereira 2007, Chaves et al. 2009, Guidi et al. 2017, Papadakis et al. 2019, Sharma et al. 2019, Landi et al. 2020). Often, stomata limitations to photosynthesis represent the first effect of the osmotic stress promoted by $\mathrm{Na}^{+}$and $\mathrm{Cl}$ 
Table 1. Nitric oxide induced changes in plant biology during salinity stress in various plants.

\begin{tabular}{lll}
\hline Effect of nitric oxide on plant biology & Plant species & Reference \\
\hline Enhanced activity of tonoplast $\mathrm{H}^{+}$-ATPase and $\mathrm{Na}^{+} / \mathrm{H}^{+}$antiporter gene & Zea mays & (Zhang et al. 2006b) \\
Stimulated root elongation and germination rate & Lupinus luteus & (Kopyra and Gwóźdź 2003) \\
Increased in viability of leaf, enhancement in productivity & Oryza sativa & (Uchida et al. 2002) \\
Enhancement in cytosolic $\mathrm{K}^{+}$content & Triticum aestivum & (Ruan et al. 2004) \\
Enhancement in proline synthesis & Lycopersicom esculentum & (Wu et al. 2011) \\
Enhanced accumulation of polyamines like spermidine & Cucumis sativus & (Fan et al. 2013) \\
$\begin{array}{l}\text { Enhanced activity of antioxidative enzymes and content of proline; } \\
\text { reduction in malondialdehyde content }\end{array}$ & Brassica juncea & (Zeng et al. 2011) \\
Decreased $\mathrm{Na}^{+}$content with simultaneous increase in $\mathrm{K}^{+}$content & Kosteletzkya virginica & (Guo et al. 2009) \\
\hline
\end{tabular}

accumulation in plant tissues (Chaves et al. 2009, Sotiras et al. 2019). In a second phase, when both ions accumulate at toxic level, photosynthesis can be further reduced by damages to chloroplast, and in particular to photosystem II (Pompeiano et al. 2017).

Nitric oxide has been demonstrated to revert the adverse effect of salinity to the photosynthetic machinery in many instances. For example, gas exchange and chlorophyll fluorescence parameters were found to be less impaired in Hordeum vulgare and Lycopersicon esculentum seedlings under saline conditions when treated with exogenous NO (Zhang et al. 2006a, Wu et al. 2011). Amelioration of photosynthesis was observed in plants subjected to salinity by the application of NO with or without sulfur (S) (Fatma et al. 2016a). However, maximal increase in photosynthesis was noted with the combined treatment of NO plus S. Nitric oxide independently or in combination with $\mathrm{S}$ promoted the synthesis of glutathione, assimilation of S, optimum production of NO and redox state, which represent the bases of NO-triggered defensive mechanism of mustard plants (Fatma et al. 2016b). Recent reports suggest that NO treatment reduced the salt toxicity by enhancing the efficiency of photosynthetic system in mustard plants (Jahan et al. 2020). It has also been demonstrated that application of NO increased photosynthesis through increase of Rubisco activity and $g_{s}$ (Fatma and Khan 2014). Nitric oxide induce the expression of the plasma membrane $\mathrm{H}^{+}$-ATPase required for a balanced $\mathrm{K}^{+}: \mathrm{Na}^{+}$ion ratio providing protection against salt stress (Zhao et al. 2004). Indeed, the maintenance of a balanced $\mathrm{K}^{+}$flux is essential to control stomata aperture under salinity. The exogenous application of NO in plants of Triticum aestivum induced stomatal opening that were partially reversed by salt stress (Sehar et al. 2019). In NOtreated plants, the lower impact on stomata closure resulted in a lower decline of $\mathrm{P}_{\mathrm{N}}$ when plants were subjected to salinity. The authors hypothesized that application of NO increased GSH content which played a role in cellular redox homeostasis and regulation of stomatal movement. GSH has also been reported to interact with ABA to regulate stomata movements (Misra et al. 2015).

NO responses also correlated with the amount of nutrients available to the plant. According to Wang et al. (2013) the improvement of the process of photosynthesis, biosynthesis of chlorophylls, and transpiration rate depended upon the availability of $\mathrm{Mg}$ and $\mathrm{Fe}$ in perennial ryegrass. Apart from this, exogenous treatment of NO along with salicylic acid also stimulated the uptake and translocation of $\mathrm{Fe}$ in Arachis hypogaea, even in the presence of salt stress (Kong et al. 2014), which could be a remarkable factor for salt-stress tolerance. The mechanisms involved in regulation of photosynthesis by NO under salt stress are shown in Fig. 1.

Regulation of reactive oxygen species and enzymatic antioxidants: Against oxidative stress, NO plays a crucial role in resisting the stress (Ahmad et al. 2016, Sharma et al. 2020). Salt stress causes changes in the assimilation pathway of nitrates and sulphates by altering the expression of various enzymes, further depressing their energy status, and enhancing nitrogen and sulfur demands. Injuries caused at cellular level are mainly due to the oxidative damage generated by ROS produced in saline conditions.

Various detoxification strategies are developed by plants growing under salinity, such as boosting up the activities of certain antioxidative enzymes like superoxide dismutase (SOD), guaiacol peroxidase (POD), catalase (CAT), ascorbate peroxidase (APOX), glutathione reductase (GR) along with certain non-enzymatic antioxidants like tocopherols, glutathiones, ascorbates which help in scavenging of free radicals (Nazar et al. 2015). Nitric oxide helps in regulation of salt induced oxidative stress and increasing resistance of plants growing in those challenging conditions. For example, when NO is applied to maize plants, reduction in the content of hydrogen peroxide was observed, accompanied by the activation of antioxidative defense system of maize that led to amelioration of free radical content and improvement in cell viability (Farooq et al. 2009). Oxidative damage caused by salt stress in Brassica juncea plants were significantly overcome by the exogenous NO which further stimulated the activities POD, SOD, APX, and GR (Zeng et al. 2011). In Oryza sativa, NO treatment significantly alleviated salt stress by modulating expression of antioxidative enzymes resulting in enhanced salt resistance (Uchida et al. 2002). The overview of mechanisms involved in regulation of oxidative stress by NO is given in Fig. 2 . 
Regulation of other metabolites: Biosynthesis of proline enhances in the cytosols with the treatment of NO. For example, in Kosteletzkya virginica plants accumulation of proline in cytosol was drastically stimulated under salt stress when SNP was applied (Guo et al. 2009). Similarly in wheat plants, free radicals were efficiently scavenged and stabilization of macromolecules occurs with enhancing the content of proline after NO treatment under salinity (Ruan et al. 2002). Additionally, NO promote accumulation of proline in coordination with sugars. For example, in Lycopersicom esculentum, application of NO resulted in enhanced proline and sugar content leading to increase in salt tolerance (Wu et al. 2011). In tomato plants retardation in the content of photosynthetic pigments were reported due to salt stress, but enhanced content of proline after NO treatment significantly ameliorated the negative impacts of salinity (Wu et al. 2010). Regulation of proline accumulation by NO under salt stress is directly related to the reduction of harmful ROS and better salt tolerance in plants (Guo et al. 2009).

Glycine-betaine (GB) plays crucial role in regulation of salt stress in plants. Nitric oxide also helps in regulating the GB mediated stress responses in salt stressed plants (Kumari et al. 2019). Further, these researchers observed that transcription and activity of betaine aldehydedehydrogenase (BADH) in light-grown seedling cotyledons were found to be very high in comparison to those seedlings which were grown in dark when facing

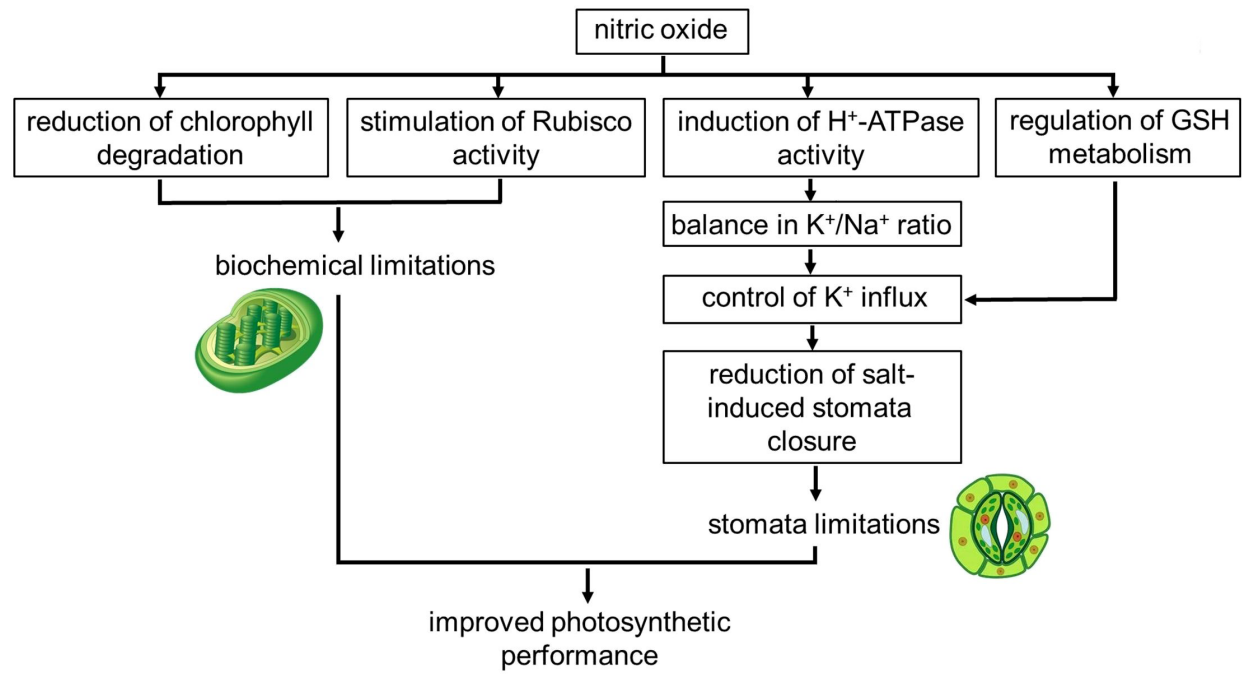

Fig. 1. An overview of nitric oxide mediated regulation of photosynthesis in plants under salt stress. GSH - glutathione.

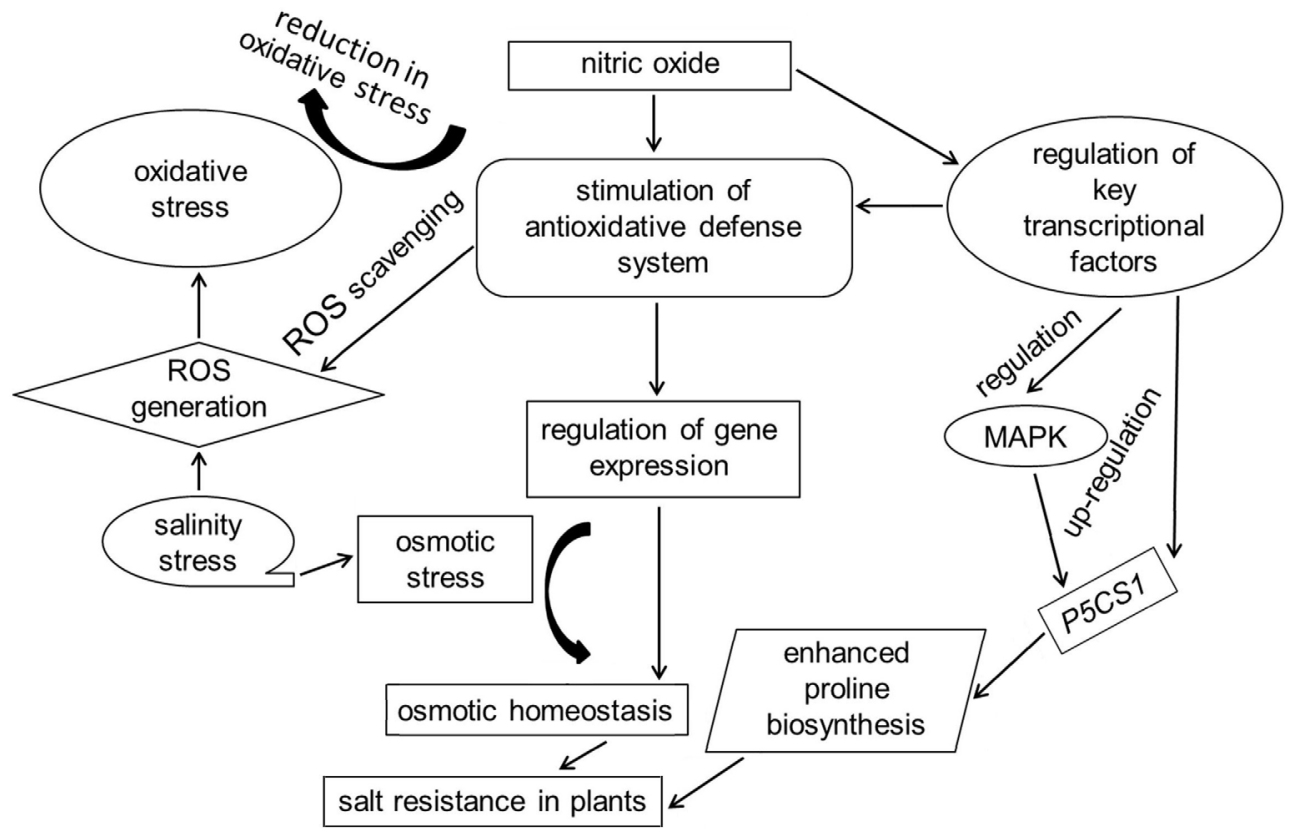

Fig. 2. A mechanism showing the alleviation of salt stress in plants by exogenous application of nitric oxide. P5CS1 - pyrroline-5carboxylate synthetase, MAPK - mitogen-activated protein kinase, ROS - reactive oxygen species. 
the salinity stress. Application of diethylenetriamine, a NO donor also contributed in maintaining the activity of $\mathrm{BADH}$, hence maintaining the homeostasis of GB. It suggests a possible crosstalk between $\mathrm{NO}$ and GB in counterattacking the salinity stress (Kumari et al. 2019). This modification in the GB content occurs after application of $\mathrm{NO}$ due to BADH transcriptional regulation or nitrosylation. Additionally, NO has potential to stimulate the synthesis of osmolytes under salinity stress (Ruan et al. 2004) and can also establish a relation between salt stress, NO application/deposition and stimulation of osmolytes like proline which further boost quenching of free radicals.

Polyamines are also involved in salt stress tolerance and their regulation by NO further improves the salinity resistance of plants. For example, in cucumber seedlings, application of SNP triggered the biosynthesis and accumulation of polyamines (Fan et al. 2013). These researchers noticed that in comparison to control plants, content of spermine, spermidine, and putrescine decrease in the salt stressed plants in dose-dependent manner. Additionally, in presence of NO, activity of polyamine oxidase was also improved accompanied by enhancing resistance to salt stress (Fan et al. 2013).

The role of $\mathrm{NO}$ in regulating leaf water content is dependent upon abscisic acid (ABA) at low concentrations of salts, whereas at high concentrations, maintenance of leaf water is done by NO mediated closing the stomatal aperture (Hua et al. 2004). For the regulation of stomatal oscillations, ABA signaling is facilitated by $\mathrm{NO}$ in guard cells. With reduction of the NO content, stomatal closure induced by ABA is retarded (Steven et al. 2002). The transpiration rate is retarded in various plants like Salpichroa organifolia and Vicia faba by the application of NO, which further causes closing stomata via ABA modulated pathways (García-Mata et al. 2001). However, when inhibitors of NO were supplied, it reverse NOinduced stomatal closure (Bright 2006).

\section{Regulation of ions by nitric oxide under salt stress}

As mentioned above, $\mathrm{NO}$ also regulates the ion balance in plants resulting in better salt stress tolerance. In Zea mays NO application resulted in improvement of the salt resistance by enhancing the $\mathrm{Na}^{+} / \mathrm{H}^{+}$antiport along with proton-pump activity of the tonoplast (Zhang et al. 2006b). In sunflower seedlings, NO modulated the biochemical responses under salt stress by regulating the ratio of $\mathrm{Na}^{+} /$ $\mathrm{K}^{+}$ions accompanied by better salt tolerance (David et al. 2010). Nitric oxide also regulates ions like $\mathrm{K}^{+}$, $\mathrm{Mg}^{2+}, \mathrm{Ca}^{2+}$ in plants facing salt stress. This NO mediated ion regulation is further accompanied by a reduction in oxidative stress and improvement of plant growth in terms of better photosynthetic rate, chlorophyll content, stomatal conductance, transpiration rate, and enhanced activities of antioxidative enzymes (Khoshbakht et al. 2018).

\section{Conclusions and future perspectives}

Though several NO-mediated mechanisms have been described in plants subjected to salt stress, and most of them are able to ameliorate plant growth and reproduction. Among others, NO reacts and interacts with other signaling compounds, describing a finely-tuned and well-orchestrated network once plants experience saline conditions. In addition, it contributes in regulating $\mathrm{H}^{+}$-ATPase thereby balancing $\mathrm{Na}^{+} / \mathrm{K}^{+}$ratio under salinity and promoting the biosynthesis of some osmolytes, which can be useful to prevent salt-triggered water stress. Nitric oxide also interacts with ROS, and has been shown to regulate salt stress responses and programmed cell death. It is conceivable that many other key aspects about this versatile compound are yet to be described.

Further researches are necessary to deepen the signaling behavior of NO in signal transduction in addition to transcriptional regulation and ion detoxification. The advancement of research focusing on NO might be helpful in understanding the involvement of this compound in plants under stress, thereby posing the bases to use NO as a possible strategy to improve salt tolerance and valorize marginal (salinized) lands. This might be helpful to both counteract soil erosion and increase the productivity of these marginal areas, in the attempt to contribute in food supply to a raising human population.

\section{References}

Acosta-Motos, J.R., Ortuño, M.F., Bernal-Vicente, A., DiazVivancos, P., Sanchez-Blanco, M.J., Hernandez, J.A.: Plant responses to salt stress: adaptive mechanisms. - Agronomy 7: 18, 2017.

Ahmad, P., Abdel Latef, A.A., Hashem, A., Abd Allah, E.F., Gucel, S., Tran, L.S.P.: Nitric oxide mitigates salt stress by regulating levels of osmolytes and antioxidant enzymes in chickpea. - Front. Plant Sci. 7: 347, 2016.

Bright, J.: Nitric oxide Signalling in Arabidopsis thaliana Guard Cells. - University of the West of England, Bristol 2006.

Bright, J., Hiscock, S.J., James, P.E., Hancock, J.T.: Pollen generates nitric oxide and nitrite: a possible link to polleninduced allergic responses. - Plant Physiol. Biochem. 47: 49$55,2009$.

Chartzoulakis, K., Klapaki, G.: Response of two greenhouse pepper hybrids to $\mathrm{NaCl}$ salinity during different growth stages. - Sci. Hort. 86: 247-260, 2000.

Chaves, M.M., Flexas, J., Pinheiro, C.: Photosynthesis under drought and salt stress: regulation mechanisms from whole plant to cell. - Ann. Bot. 103: 551-560, 2009.

Chinnusamy, V., Zhu, J., Zhu, J.K.: Salt stress signaling and mechanisms of plant salt tolerance. - In Setlow, J.K. (ed.): Genetic Engineering. Vol. 27. Pp. 141-177. Springer,Boston 2006.

David, A., Yadav, S., Bhatla, S.C.: Sodium chloride stress induces nitric oxide accumulation in root tips and oil body surface accompanying slower oleosin degradation in sunflower seedlings. - Physiol. Plant. 140: 342-354, 2010.

Fan, H.F., Du, C.X., Guo, S.R.: Nitric oxide enhances salt 
tolerance in cucumber seedlings by regulating free polyamine content. - Environ. exp. Bot. 86: 52-59, 2013.

Fancy, N.N., Bahlmann, A.K., Loake, G.J.: Nitric oxide function in plant abiotic stress. - Plant Cell Environ. 40: 462-472, 2017.

Farooq, M., Basra, S.M.A., Wahid, A., Rehman, H.: Exogenously applied nitric oxide enhances the drought tolerance in fine grain aromatic rice (Oryza sativa L.). - J. Agron. Crop Sci. 195: 254-261, 2009

Fatma, M., Khan, N.A.: Nitric oxide protects photosynthetic capacity inhibition by salinity in Indian mustard. - J. funct. environ. Bot. 4: 106-116, 2014

Fatma, M., Masood, A., Per, T.S., Khan, N.A.: Nitric oxide alleviates salt stress inhibited photosynthetic performance by interacting with sulfur assimilation in mustard. - Front. Plant Sci. 7: 521, 2016a

Fatma, M., Masood, A., Per, T.S., Rasheed, F., Khan, N.A.: Interplay between nitric oxide and sulfur assimilation in salt tolerance in plants. - Crop J. 4: 153-161, 2016b.

Ferrer, M.A., Ros Barceló, A.: Differential effects of nitric oxide on peroxidase and $\mathrm{H}_{2} \mathrm{O}_{2}$ production by the xylem of Zinnia elegans. - Plant Cell Environ. 22: 891-897, 1999.

Gadelha, C.G., De Souza Miranda, R., Alencar, N.L.M., Costa, J.H., Prisco, J.T., Gomes-Filho, E.: Exogenous nitric oxide improves salt tolerance during establishment of Jatropha curcas seedlings by ameliorating oxidative damage and toxic ion accumulation. - J. Plant Physiol. 212: 69-79, 2017.

García-Mata, C., Lamattina, L.: Nitric oxide induces stomatal closure and enhances the adaptive plant responses against drought stress. - Plant Physiol. 126: 1196-1204, 2001.

Guidi, L., Remorini, D., Cotrozzi, L., Giordani, T., Lorenzini, G., Massai, R., Nali, C., Natali, L., Pellegrini, E., Trivellini, A.: The harsh life of an urban tree: the effect of a single pulse of ozone in salt-stressed Quercus ilex saplings. - Tree Physiol. 37: 246-260, 2017.

Guo, F.Q., Okamoto, M., Crawford, N.M.: Identification of a plant nitric oxide synthase gene involved in hormonal signaling. - Science 302: 100-103, 2003.

Guo, Y., Tian, Z., Yan, D., Zhang, J., Qin, P.: Effects of nitric oxide on salt stress tolerance in Kosteletzkya virginica. - Life Sci. J. 6: 67-75, 2009.

Hanin, M., Ebel, C., Ngom, M., Laplaze, L., Masmoudi, K.: New insights on plant salt tolerance mechanisms and their potential use for breeding. - Front. Plant Sci. 7: 1787, 2016.

Hasanuzzaman, M., Oku, H., Nahar, K., Bhuyan, M.B., Al Mahmud, J., Baluska, F. Fujita, M.: Nitric oxide-induced salt stress tolerance in plants: ROS metabolism, signaling, and molecular interactions. - Plant Biotechnol. Rep. 12: 77-92, 2018.

He, Y., Tang, R.H., Hao, Y., Stevens, R.D., Cook, C.W., Ahn, S.M., Jing, L., Yang, Z., Chen, L., Guo, F., Fiorani, F.: Nitric oxide represses the Arabidopsis floral transition. - Science 305: 1968-1971, 2004.

Hernández, J.A., Campillo, A., Jiménez, A., Alarcón, J.J., Sevilla, F.: Response of antioxidant systems and leaf water relations to $\mathrm{NaCl}$ stress in pea plants. - New Phytol. 141: 241-251, 2002.

Hua, X., Tan, L., An, L., Zhao, Z., Wang, S., Zhang, C.: Evidence for the involvement of nitric oxide and reactive oxygen species in osmotic stress tolerance of wheat seedlings: Inverse correlation between leaf abscisic acid accumulation and leaf water loss. - Plant Growth Regul. 42: 61-68, 2004.

Jahan, B., Al Ajmi, M. F., Rehman, M. T., Khan, N.A.: Treatment of nitric oxide supplemented with nitrogen and sulfur regulates photosynthetic performance and stomatal behavior in mustard under salt stress. - Physiol. Plant. 168: 490-510, 2020.

Khavari-Nejad, R.A., Mostofi, Y.: Effects of $\mathrm{NaCl}$ on photosynthetic pigments, saccharides, and chloroplast ultrastructure in leaves of tomato cultivars. - Photosynthetica 35: 151-154, 1998.

Khoshbakht, D., Asghari, M.R., Haghighi, M.: Effects of foliar applications of nitric oxide and spermidine on chlorophyll fluorescence, photosynthesis and antioxidant enzyme activities of citrus seedlings under salinity stress. - Photosynthetica 56: 1313-1325, 2018.

Kong, J., Dong, Y., Xu, L., Liu, S., Bai, X.: Effects of foliar application of salicylic acid and nitric oxide in alleviating iron deficiency induced chlorosis of Arachis hypogaea L. - Bot. Stud. 55: 9, 2014.

Kopyra, M., Gwóźdź, E.A.: Nitric oxide stimulates seed germination and counteracts the inhibitory effect of heavy metals and salinity on root growth of Lupinus luteus. - Plant Physiol. Biochem. 41: 1011-1017, 2003.

Kumari, A., Kapoor, R., Bhatla, S.C.: Nitric oxide and light coregulate glycine betaine homeostasis in sunflower seedling cotyledons by modulating betaine aldehyde dehydrogenase transcript levels and activity. - Plant Signal Behav. 14: 1666656, 2019.

Landi, M., Araniti, F., Flamini, G., Piccolo, E.L., Trivellini, A., Abenavoli, M.R., Guidi, L.: "Help is in the air": volatiles from salt-stressed plants increase the reproductive success of receivers under salinity. - Planta 251: 1-15, 2020.

Misra, B.B., Acharya, B.R., Granot, D., Assmann, S.M., Chen, S.: The guard cell metabolome: functions in stomatal movement and global food security. - Front. Plant Sci. 6: 334, 2015.

Munns, R.: Comparative physiology of salt and water stress. Plant cell Environ. 25: 239-250, 2002.

Mur, L.A.J., Carver, T.L.W., Prats, E.: NO way to live; the various roles of nitric oxide in plant-pathogen interactions. J. exp. Bot. 57: 489-505, 2005.

Nazar, R., Umar, S., Khan, N.A.: Exogenous salicylic acid improves photosynthesis and growth through increase in ascorbate-glutathione metabolism and $\mathrm{S}$ assimilation in mustard under salt stress. - Plant Signal. Behav. 10: e1003751, 2015.

Neill, S., Barros, R., Bright, J., Desikan, R., Hancock, J., Harrison, J., Morris, P., Ribeiro, D., Wilson, I.: Nitric oxide, stomatal closure, and abiotic stress. - J. exp. Bot. 59: 165-176, 2008.

Papadakis, I., Sotiras, M.I., Landi, M., Ladikou, E., Oikonomou, A., Psychoyou, M., Fasseas, C.: Salinity alters plant's allometry and sugar metabolism, and impairs the photosynthetic process and photosystem II efficiency in Eriobotrya japonica plants. Agrochimica 63: 27-42, 2019.

Parida, A.K., Das, A.B.: Salt tolerance and salinity effects on plants: a review. - Ecotoxicol. Environ. Safety 60: 324-349, 2005.

Pompeiano, A., Landi, M., Meloni, G., Vita, F., Guglielminetti, L., Guidi, L.: Allocation pattern, ion partitioning, and chlorophyll a fluorescence in Arundo donax L. in responses to salinity stress. - Plant Biosystems 151: 613-622, 2017.

Rattan, A., Kapoor, D., Kapoor, N., Bhardwaj, R., Sharma, A.: Brassinosteroids regulate functional components of antioxidative defense system in salt stressed maize seedlings. - J. Plant Growth Regul. 1-11, 2020.

Ruan, H.H., Shen, W.B., Xu, L.L.: Nitric oxide involved in the abscisic acid induced proline accumulation in wheat seedling leaves under salt stress. - Acta bot. sin. 46: 1307-1315, 2004.

Ruan, H., Shen, W., Ye, M., Xu, L.: Protective effects of nitric oxide on salt stress-induced oxidative damage to wheat (Triticum aestivum L.) leaves. - Chin, Sci. Bull. 47: 677-681, 2002.

Rui, M., Ricardo, S.: Soil salinity: effect on vegetable crop growth. Management practices to prevent and mitigate soil 
salinization. - Horticulturae 3: 30, 2017.

Sehar, Z., Masood, A., Khan, N.A.: Nitric oxide reverses glucosemediated photosynthetic repression in wheat (Triticum aestivum L.) under salt stress. - Environ. exp. Bot. 161: 277 $289,2019$.

Sharma, A., Kumar, V., Shahzad, B., Ramakrishnan, M., Sidhu, G.P.S., Bali, A.S., Handa, N., Kapoor, D., Yadav, P., Khanna, K.: Photosynthetic response of plants under different abiotic stresses: a review. - J. Plant Growth Regul. 1-23, 2019.

Sharma, A., Soares, C., Sousa, B., Martins, M., Kumar, V., Shahzad, B., Sidhu, G.P., Bali, A.S., Asgher, M., Bhardwaj, R.: Nitric oxide mediated regulation of oxidative stress in plants under metal stress: a review on molecular and biochemical aspects. - Physiol. Plant. 168: 318-344. 2020.

Sotiras, M., Papadakis, I., Landi, M., Tsaniklidis, G., Tsiantas, P., Psychoyou, M.: Allocation pattern, photosynthetic performance and sugar metabolism in hydroponically grown seedlings of loquat (Eriobotrya japonica Lindl.) subjected to salinity. - Photosynthetica 57: 258-267, 2019.

Tanveer, M., Shahzad, B., Sharma, A., Biju, S., Bhardwaj, R.: 24-Epibrassinolide; an active brassinolide and its role in salt stress tolerance in plants: a review. - Plant Physiol. Biochem. 130: 69-79, 2018.

Teixeira, J., Pereira, S.: High salinity and drought act on an organdependent manner on potato glutamine synthetase expression and accumulation. - Environ. exp. Bot. 60: 121-126, 2007.

Uchida, A., Jagendorf, A.T., Hibino, T., Takabe, T., Takabe, T.: Effects of hydrogen peroxide and nitric oxide on both salt and heat stress tolerance in rice. - Plant Sci. 163: 515-523, 2002.

Vengosh, A.: Salinization and saline environments. - In Holland, H.D. Turekian, K.K. (ed.): Treatise on Geochemistry. Vol. 9. Pp 325-378. Elsevier, Oxford 2003.

Wang, Q., Liang, X., Dong, Y., Xu, L., Zhang, X., Hou, J., Fan, $Z$ : : Effects of exogenous nitric oxide on cadmium toxicity, element contents and antioxidative system in perennial ryegrass. - Plant Growth Regul. 69: 11-20, 2013.

Wang, Y., Nii, N.: Changes in chlorophyll, ribulose bisphosphate carboxylase-oxygenase, glycine betaine content, photosynthesis and transpiration in Amaranthus tricolor leaves during salt stress. - J. hort. Sci. Biotechnol. 75: 623-627, 2000.

Wu, X., Zhu, W., Zhang, H., Ding, H., Zhang, H.J.: Exogenous nitric oxide protects against salt-induced oxidative stress in the leaves from two genotypes of tomato (Lycopersicom esculentum Mill.). - Acta Physiol. Plant. 33: 1199-1209, 2011.

Wu, X.X., Ding, H.D., Chen, J.L., Zhang, H.J., Zhu, W.M.: Attenuation of salt-induced changes in photosynthesis by exogenous nitric oxide in tomato (Lycopersicon esculentum Mill. L.) seedlings. - Afr. J. Biotechnol. 9: 7837-7846, 2010.

Yemets, A.I., Krasylenko, Y.A., Sheremet, Y.A., Blume, Y.B.: Microtubule reorganization as a response to realization of no (II) signals in plant cell. - Tsitol. Genet. 43: 3-10, 2009.

Zeng, C.L., Liu, L., Wang, B.R., Wu, X.M., Zhou, Y.: Physiological effects of exogenous nitric oxide on Brassica juncea seedlings under $\mathrm{NaCl}$ stress. - Biol. Plant. 55: 345$348,2011$.

Zhang, L., Wang, Y., Zhao, L., Shi, S., Zhang, L.: Involvement of nitric oxide in light-mediated greening of barley seedlings. - J. Plant Physiol. 163: 818-826, 2006 a.

Zhang, Y., Wang, L., Liu, Y., Zhang, Q., Wei, Q., Zhang, W.: Nitric oxide enhances salt tolerance in maize seedlings through increasing activities of proton-pump and $\mathrm{Na}^{+} / \mathrm{H}^{+}$ antiport in the tonoplast. - Planta 224: 545-555, 2006 b.

Zhao, L., Zhang, F., Guo, J., Yang, Y., Li, B., Zhang, L.: Nitric oxide functions as a signal in salt resistance in the calluses from two ecotypes of reed. - Plant Physiol. 134: 849-857, 2004.

Zhao, M.G., Tian, Q.Y., Zhang, W.H.: Nitric oxide synthasedependent nitric oxide production is associated with salt tolerance in Arabidopsis. - Plant Physiol. 144: 206-217, 2007.

Li, Q., Lv, R.L., Teng, Z.J., Si, L.B., Ma, T., Yang, Y.T.: Apoplastic hydrogen peroxide and superoxide anion exhibited different regulatory functions in salt-induced oxidative stress in wheat leaves. - Biol. Plant. 62: 750-762, 2018. 